\title{
Spotiton and Chameleon Vitrification robots
}

\author{
Hui Wei ${ }^{1}$, Venkata P. Dandey ${ }^{1}$, Mykhailo Kopylov' ${ }^{1}$ Edward T. Eng ${ }^{1}$, Peter Kahn², Clinton S. Potter ${ }^{1,3}$, \\ Bridget Carragher ${ }^{1,3}$
}

1. National Resource for Automated Molecular Microscopy, Simons Electron Microscopy Center, New York Structural Biology Center, New York, USA.

2. Engineering Arts LLC, Tempe, AZ, USA.

3. Department of Biochemistry and Molecular Biophysics, Columbia University, New York, NY, USA.

Almost every aspect of cryo electron microscopy (cryoEM) has been automated over the last few decades. One of the challenges that remains to be addressed is the robust and reliable preparation of vitrified specimens of suitable ice thickness. To this end we have been developing a new approach that uses piezo dispensing of picoliter volumes coupled to "self-blotting" nanowire grids $[3,4]$ to vitrify very small amounts of sample without the use of externally applied blotting paper [2]. The prototype instrument (Spotiton) [1] is now being developed as a commercial system (Chameleon, TTP LabTech Ltd.) which has the potential to provide a robust and highly automated method for making vitrified grids. This approach to vitrification also the additional advantage that it results in shorter dwell times of the sample in the thin liquid film just prior to vitrification and this appears to ameliorate some of the deleterious effects of the sample interacting with the air-water interface.

Chameleon (Fig 1a and 1b) features, as of February, 2019, include: (a) Aspiration from sample volumes as small as $\sim 3 \mu$ l, sufficient to vitrify hundreds of grids. (b) Once vitrified, grids are automatically transferred into grid boxes for storage. (c) Optical images of the sample on the grid are collected $50 \mathrm{~ms}$ before plunging into ethane which is useful to assess ice thickness without loading into a TEM. (d) Auto generation of vitrification reports including instrument and environmental parameters and grid box location. Future improvements should include: (a) Temperature controlled sample block. (b) Multiple grids loaded into the instrument prior to vitrification which reduces manual operation to a single grid handling step. (c) Faster dispense-to-plunge times $(<100 \mathrm{~ms})$. (d) Cryogen stability and control to remove the need to visually check cryogens. Using an experimental prototype of Chameleon, we have been able to vitrify a wide variety of samples including our usual roster of test samples (Apoferritin, 20S proteasome, ribosomes, Adeno-associated virus serotype 2 (AAV2)) as well as many user samples. Figures 2 shows near atomic resolution structures of Apoferritin from grids vitrified by Chameleon. We will present a variety of other examples from user projects that illustrate the usefulness of this approach in reducing the negative effects of the air-water interface.

We have also recently been exploring using Spotiton technology for "time resolved" mixing of samples. We have added a second independent piezo dispense head to Spotiton. Each head can fire independently (figure 3) but be targeted to deposit sample almost simultaneously (within 4 msecs) to the same location on the grid. Preliminary results show that we can effectively and efficiently mix two samples within a few milliseconds and then freeze the process about 100 msecs later. We are currently exploring the utility of this system for trapping time resolved biological mechanisms and these results will be presented [5].

\section{References:}

[1] Dandey et al., J Struct Biol. 2018.

[2] Razinkov et al., J Struct Biol. 195 (2016) p. 190.

[3] Wei et al., J Struct Biol. 2018.

[4] F Zhang et al., Adv. Mater. 25 (2013), p. 4192. 
[5] Some of this work was performed at the Simons Electron Microscopy Center and National Resource for Automated Molecular Microscopy located at the New York Structural Biology Center, supported by grants from the Simons Foundation (349247), NYSTAR, and the NIH (GM103310, RR029300).

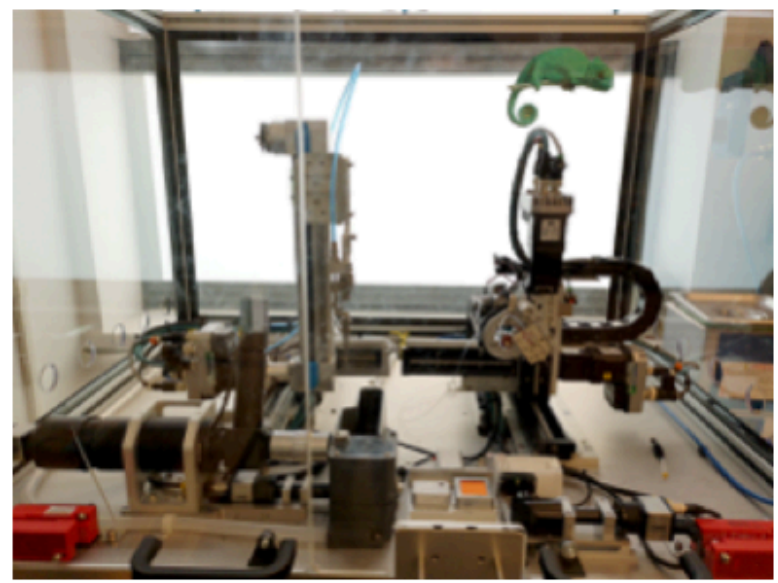

Fig 1: Prototype of Chameleon robot.
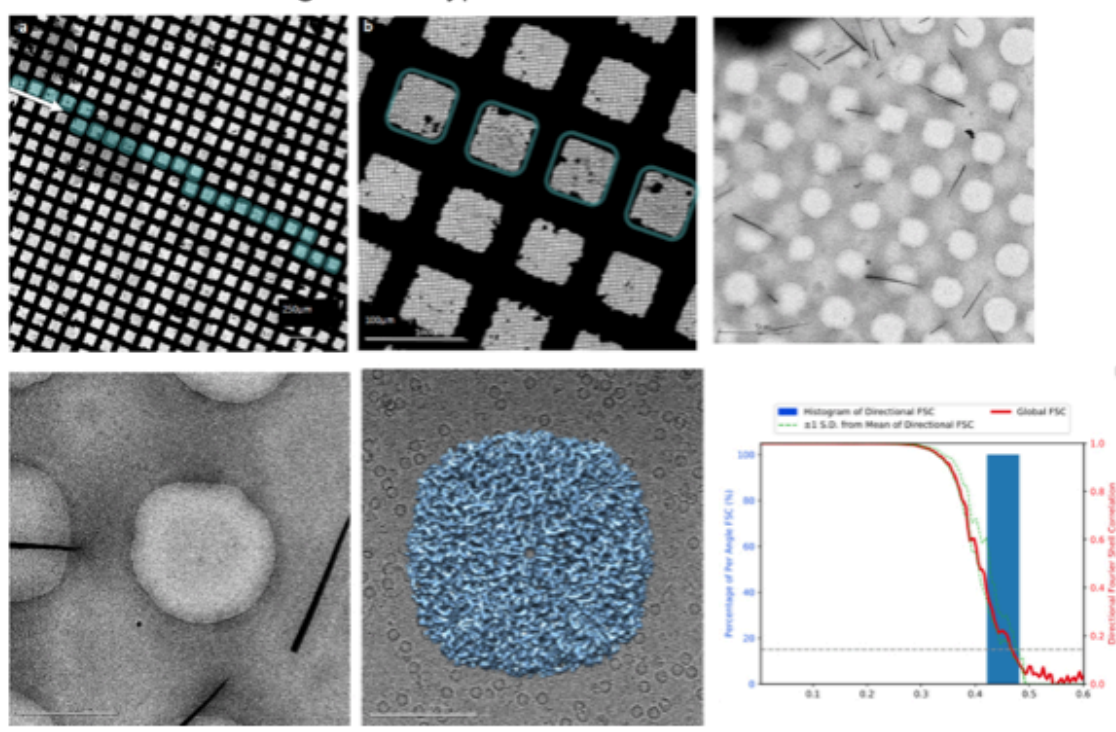

Fig 2: 2.2 A map of Apoferritin; sample vitrified by the Chameleon robot.
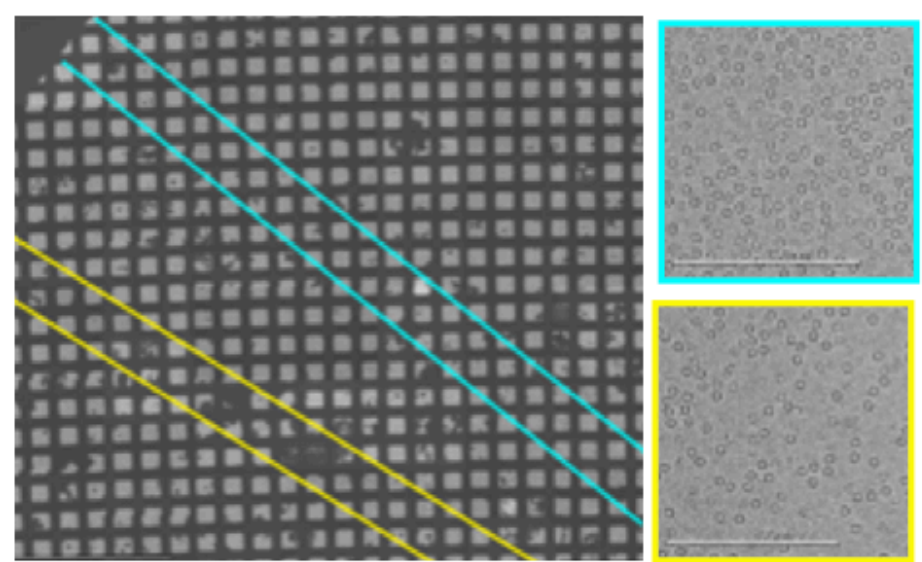

Fig 3: Two separate heads have been installed on Spotiton, shown here dispensing $2 \mathrm{mg} . \mathrm{ml}$ (blue) and $4 \mathrm{mg} / \mathrm{ml}$ (yellow) samples in separate stripes. The stripes can be overlaid to mix two samples together on the grid. 